\title{
SPLITTING STRONGLY ALMOST DISJOINT FAMILIES
}

\author{
A. HAJNAL, I. JUHÁSZ AND S. SHELAH
}

\begin{abstract}
We say that a family $A \subset[\lambda]^{\kappa}$ is strongly almost disjoint if something more than just $|A \cap B|<\kappa$, e.g. that $|A \cap B|<\sigma<\kappa$, is assumed for $A, B \in A$. We formulate conditions under which every such strongly a.d. family is "essentially disjoint", i.e. for each $A \in A$ there is $F(A) \in[A]^{<\kappa}$ so that $\{A \backslash F(A): A \in A\}$ is disjoint. On the other hand, we get from a supercompact cardinal the consistency of $\mathrm{GCH}$ plus the existence of a family $A \subset\left[\omega_{\omega+1}\right]^{\omega_{1}}$ whose elements have pairwise finite intersections and such that it does not even have property $B$. This solves an old problem raised in [4]. The same example is also used to produce a graph of chromatic number $\omega_{2}$ on $\omega_{\omega+1}$ that does not contain $[\omega, \omega]$, answering a problem from [5].

We also have applications of our results to "splitting" certain families of closed subsets of a topological space. These improve results from $[3,12$ and 13].
\end{abstract}

0. Introduction. One of the oldest results of set theory applied to topology is Bernstein's theorem [2] which says that the real line $\mathbf{R}$ can be split into two disjoint parts, say $X_{0}$ and $X_{1}$, in such a way that every Cantor set in $\mathbf{R}$ (or, equivalently, every uncountable closed subset of $\mathbf{R}$ ) intersects both $X_{0}$ and $X_{1}$. Using the terminology introduced in [8], this means that the family $C(\mathbf{R})$ of all Cantor sets in $\mathbf{R}$ has property $B$.

There is another, by now classical, result due to Miller [8] which states that if $A$ is any strongly almost disjoint family of countably infinite sets (here "strongly almost disjoint" means that there is a $n \in \omega$ such that if $A, B \in A$ and $A \neq B$ then $|A \cap B|<n)$, then $A$ has property $B$.

In this paper we propose to show that both of these classical results, as well as numerous further strengthenings and generalizations of them, boil down to the same thing. More precisely, we will show that all of these results (see e.g. $[\mathbf{3}, \mathbf{4}$, $\mathbf{7}, \mathbf{8}, \mathbf{1 2}, \mathbf{1 3}])$ are relatively easy consequences of a single general theorem that we call the Main Theorem. It should not come then as a surprise that such a general result has a somewhat complicated formulation.

The two classical results we mentioned above are, of course, proved in ZFC, but some of the strengthenings and/or generalizations did require assumptions going beyond ZFC. We shall also present here several results that show that some of these extra set-theoretic hypotheses are indeed essential. Thus we shall solve problems raised to this effect, e.g., in [4].

1. The Main Theorem. Our set-theoretic notation is standard. We shall list below some (really simple) definitions, notations and terminology that we need to give a transparent formulation of our Main Theorem.

Received by the editors June 11, 1985.

1980 Mathematics Subject Classification. Primary 04A20, 04A30, 03E35, 03E55; Secondary 54A25, 54A35. 
Given a set $X$, a family of subsets of $X$, say $J$, is said to be a semi-ideal on $X$ if $B \subset A \in J$ implies $B \in J$, i.e. $J$ is downward hereditary. An ideal on $X$ is a semi-ideal that is also closed under finite unions. An ideal is said to be $\kappa$-additive (for some infinite cardinal $\kappa$ ) if it is closed under unions of subfamilies of size $<\kappa$. (Thus every ideal is $\omega$-additive.)

For any $A \subset P(X)$ we let $\hat{A}$ denote the semi-ideal generated by $A$, i.e.

$$
\hat{A}=\{B \subset X: \exists A \in A \text { s.t. } B \subset A\} .
$$

Moreover, if $I$ is any (semi-)ideal on $X$ then we define the $I$-restriction of $A$ to a subset $Y \subset X$ (in symbols $A \uparrow_{I} Y$ ) as follows:

$$
A \uparrow_{I} Y=\{A \cap Y: A \backslash Y \in I \& A \in A\} \text {. }
$$

Semi-ideals are families of sets that could be thought of as small in some sense. Thus if $J \subset P(X)$ is a semi-ideal on $X$ we shall put

$$
J^{+}=P(X) \backslash J .
$$

The elements of $J^{+}$are the sets which have " $J$-positive measure". Also, a family $A \subset J^{+}$is said to be $J$-almost disjoint (in short $J$-a.d.) if $A \cap B \in J$ holds for any two members $A, B \in A$.

If $\sigma$ is a (finite or infinite) cardinal then $J=[X]^{<\sigma}$ is clearly a semi-ideal on $X$. A family which is $J$-a.d. for this $J$ is called $\sigma$-a.d.

If $\mathcal{H}$ is any family of sets (and $\tau$ is a fixed cardinal) then $U \mathcal{H}\left(\mathcal{U}_{<\tau} \mathcal{H}\right)$ denotes the family of all possible unions of increasing chains (of length less than $\tau$ ) consisting of elements of $\mathcal{H}$.

If $\Phi \subset P(P(X))$ then we say that $\Phi$ is a property of families of subsets of $X$ and instead of $A \in \Phi$ we usually write $\Phi(A)$. Now if $I$ is an ideal on $X$ then the property $\Phi$ is said to be $I$-hereditary if, for any $A \subset P(X)$ and $Y \subset X, \Phi(A)$ implies $\Phi\left(A \uparrow_{I} Y\right)$. Finally, $\Phi$ is said to be $I$-additive if the following holds: Suppose $S$ is a disjoint subfamily of $P(X)$ and $A \subset P(X)$ is such that for every $A \in A$ there is $S \in S$ with $A \backslash S \in I$. Then if $\Phi\left(\mathcal{A} \uparrow_{I} S\right)$ holds for each $S \in S$ so does $\Phi(\mathcal{A})$.

After these preliminaries we formulate a lemma that will be instrumental in the proof of the Main Theorem.

1.1. MAIN Lemma. Suppose $\kappa \geq \omega, X$ is a set with $|X|=\lambda>\kappa, I$ is an ideal and $J$ is a semi-ideal on $X$; moreover $A \subset[X]^{\leq \kappa} \cap I^{+}$and $\Phi \subset P^{2}(X)$ are such that

(1) $\hat{A} \cap(\cup J) \subset I$;

(2) there is a set of infinite cardinals $C \subset \lambda$ cofinal in the set of all cardinals preceding $\lambda$ such that for every $\gamma \in C$ and every $Y \in[X] \leq \gamma$

$$
\left|\left\{A \in A: A \cap Y \in J^{+}\right\}\right| \leq \gamma
$$

(3) $\Phi$ is I-additive and for every $Y \in[X]^{<\lambda}$ we have $\Phi\left(\mathcal{A} \uparrow_{I} Y\right)$. Then $\Phi(\mathcal{A})$ is valid.

PROOF. Let us start by writing

$$
X=\left\{x_{\alpha}: \alpha \in \lambda\right\} .
$$

Next, for any ordinal $\alpha \in \lambda$ we put, using (2),

$$
\gamma(\alpha)=\min \{\gamma \in C:|\alpha| \leq \gamma\} .
$$


Then we define sets $Y_{\alpha} \subset X$ by transfinite recursion as follows: If $\alpha$ is limit then we put

$$
Y_{\alpha}=\bigcup\left\{Y_{\beta}: \beta \in \alpha\right\}
$$

If $\alpha=\beta+1$, then we put

$$
Y_{\beta+1}=Y_{\alpha}=Y_{\beta} \cup\left\{x_{\beta}\right\} \cup \bigcup\left\{A \in A: A \cap Y_{\beta} \in J^{+}\right\} \text {. }
$$

One shows by an easy induction, using (2) again, that we have $\left|Y_{\alpha}\right| \leq \gamma(\alpha)<\lambda$ for all $\alpha \in \lambda$. It is also obvious that $\bigcup\left\{Y_{\alpha}: \alpha \in \lambda\right\}=X$. Now, let us put for each $\alpha \in \lambda$

$$
Z_{\alpha}=Y_{\omega \cdot(\alpha+1)} \backslash Y_{\omega \cdot \alpha}
$$

Then clearly $\left\{Z_{\alpha}: \alpha \in \lambda\right\}$ is a disjoint decomposition of $X$ with $\left|Z_{\alpha}\right|<\lambda$ for all $\alpha$. Thus we have, by (3), $\Phi\left(A \uparrow_{I} Z_{\alpha}\right)$ for each $\alpha$. Consequently, since $\Phi$ is $I$-additive, $\Phi(A)$ is valid if we can show that for each $A \in A$ there is some $\alpha \in \lambda$ such that $A \backslash Z_{\alpha} \in I$.

To this end, let us first note that since $A$ is the increasing union of the $A \cap Y_{\alpha}$ 's and $A \in I^{+}$, by (1) there is some $\alpha \in \lambda$ such that $A \cap Y_{\alpha} \in J^{+}$. Let $\alpha_{0}$ be minimal with this property and assume that $\omega \cdot \beta \leq \alpha_{0}<\omega \cdot(\beta+1)$. Then for each $\alpha<\omega \cdot \beta$ we have $A \cap Y_{\alpha} \in J$; consequently, using (1) again, we have

$$
A \cap Y_{\omega \cdot \beta}=\bigcup\left\{A \cap Y_{\alpha}: \alpha<\omega \cdot \beta\right\} \in I .
$$

On the other hand, since $A \cap Y_{\alpha_{0}} \in J^{+}$we have $A \subset Y_{\alpha_{0}+1} \subset Y_{\omega \cdot(\beta+1)}$. Hence, indeed,

$$
A \backslash Z_{\beta}=A \backslash\left(Y_{\omega \cdot(\beta+1)} \backslash Y_{\omega \cdot \beta}\right)=A \cap Y_{\omega \cdot \beta} \in I .
$$

In order to facilitate the formulation of our main result and several of its consequences we introduce the following technical definition.

1.2. DEFINITION. Let $\sigma$ and $\kappa$ be cardinals satisfying $\kappa=\kappa^{\sigma} \geq \omega$. Then a quintuple $\langle X, I, A, \mathcal{K}, \Phi\rangle$ is said to be $(\sigma, \kappa)$-good provided that $I$ is a $\sigma^{+}$-complete ideal on $X, A \subset[X]^{\leq \kappa} \cap I^{+}, K$ is a semi-ideal on $X, \Phi$ is a property of families of subsets of $X$ and the following conditions (i)-(iv) are satisfied:

(i) $K \cap \hat{A} \subset I$;

(ii) $A$ is $K$-a.d.;

(iii) for each $Y \in \mathcal{K}^{+} \cap \hat{A}$ there is a $Z \in[Y] \leq \sigma \cap \mathcal{K}^{+}$;

(iv) $\Phi$ is $I$-additive, $I$-hereditary and $\Phi(B)$ is valid for each $B \in[A] \leq \kappa$.

Now, our Main Theorem will be of the following form: If $\langle X, I, \mathcal{A}, \mathcal{K}, \Phi\rangle$ is $(\sigma, \kappa)$ good then $\Phi(A)$ holds, provided that some further set-theoretic assumptions are also satisfied. Our next definitions are needed for the formulation of these assumptions.

1.3. Definition. Let $\sigma, \kappa, X, I, A$ and $K$ be as in 1.2. We say that condition $A(\sigma, \kappa ; X, I, \mathcal{A}, \mathcal{K})$ holds if for every cardinal $\mu$ with $\kappa<\mu<|X|$ and $\operatorname{cf}(\mu) \leq \sigma$ there exists a cardinal $\tau>\sigma$ and a semi-ideal $J$ on $X$ such that

(a) $\min \left\{\mu^{\sigma},|X|\right\} \leq \mu^{+\tau}$ (= the $\tau$ th successor of $\mu$ );

(b) $\hat{A} \cap K \subset J$;

(c) $\hat{A} \cap \hat{U}_{<\tau} J \subset J$;

(d) $\hat{A} \cap \cup J \subset I$. of $\square$.

Next we define a combinatorial principle which, as we shall see, is a weak form 
1.4. Definition. Let $\sigma, \kappa, \mu$ be cardinals. Then $\operatorname{Sp}(\sigma, \kappa, \mu)$ denotes the following statement: There exists a sequence $\left\langle P_{\alpha}: \alpha \in \mu^{+}\right\rangle$such that for all $\alpha \in \mu^{+}$we have $P_{\alpha} \subset[\alpha]^{\sigma}$ with $\left|P_{\alpha}\right| \leq \mu$; moreover if $\alpha \in \mu$ with $\sigma<\operatorname{cf}(\alpha) \leq \kappa$ and $A$ is a cofinal subset of $\alpha$ of cardinality $\leq \kappa$, then $A$ can be written in the form $A=\bigcup\left\{A_{\nu}: \nu \in \sigma\right\}$ where for each $\nu \in \sigma$ we have $\left[A_{\nu}\right]^{\sigma} \subset \bigcup_{\beta \in \alpha} P_{\beta}$. If $\kappa=\sigma^{+}$ then instead of $\operatorname{Sp}\left(\sigma, \sigma^{+}, \mu\right)$ we simply write $\operatorname{Sp}(\sigma, \mu)$.

The following assumption to be used in our Main Theorem involves $\operatorname{Sp}(\sigma, \kappa, \mu)$.

1.5. Definition. Let $\sigma, \kappa$ and $\lambda$ be cardinals. Then $B(\sigma, \kappa, \lambda)$ denotes the following statement: $\kappa^{\sigma}=\kappa$ and for every cardinal $\mu$ if $\kappa<\mu<\lambda$ and $\operatorname{cf}(\mu) \leq \sigma$ then $\mu^{\sigma}=\mu^{+}$and $\operatorname{Sp}(\sigma, \kappa, \mu)$ holds. We write $B(\sigma, \kappa)$ to denote that $B(\sigma, \kappa, \lambda)$ is valid for all cardinals $\lambda$.

We are now ready to formulate and prove our main result:

1.6. MAIN THEOREM. Let $\sigma, \kappa$ be cardinals satisfying $\kappa^{\sigma}=\kappa$ and suppose that $\langle X, I, \mathcal{A}, \mathcal{K}, \Phi\rangle$ is $(\sigma, \kappa)$-good. Then $\Phi(\mathcal{A})$ is valid provided that either

(i) $A(\sigma, \kappa ; X, I, \mathcal{A}, \mathcal{K})$ is valid or

(ii) $B(\sigma, \kappa,|X|)$ holds.

In the proof of 1.6 we are going to make use of the following lemma.

1.7. LEMMA. If $\langle X, I, A, \mathcal{K}, \Phi\rangle$ is $(\sigma, \kappa)$-good and $X^{\prime}$ is any subset of $X$ then, putting $I^{\prime}=I \cap P\left(X^{\prime}\right), A^{\prime}=A \uparrow_{I} X^{\prime}, K^{\prime}=K \cap P\left(X^{\prime}\right)$ and $\Phi^{\prime}=\Phi \cap P^{2}\left(X^{\prime}\right)$, we have that $\left\langle X^{\prime}, I^{\prime}, \mathcal{A}^{\prime}, \mathcal{K}^{\prime}, \Phi^{\prime}\right\rangle$ is also $(\sigma, \kappa)$-good. (Note that $\mathcal{A}^{\prime}$ is not defined as $A \cap P\left(X^{\prime}\right)$.)

The straightforward (and somewhat tedious) checking required to show that all the conditions of Definition 1.2 are satisfied by $\left\langle X^{\prime}, I^{\prime}, A^{\prime}, K^{\prime}, \Phi^{\prime}\right\rangle$ is left to the reader.

PROOF OF 1.6. Assume, indirectly, that for some fixed $\sigma$ and $\kappa$ there is a $(\sigma, \kappa)$ good quintuple $\langle X, I, \mathcal{A}, \mathcal{K}, \Phi\rangle$ such that $\Phi(\mathcal{A})$ fails though either $A(\sigma, \kappa ; X, I, \mathcal{A}, \mathcal{K})$ or $B(\sigma, \kappa,|X|)$ holds true. We may, of course, assume that $|X|=\lambda$ is minimal among all such quintuples. Let us now consider any $X^{\prime} \subset X$. Then, by 1.7, the quintuple $\left\langle X^{\prime}, I^{\prime}, A^{\prime}, K^{\prime}, \Phi^{\prime}\right\rangle$ is $(\sigma, \kappa)$-good (with $I^{\prime}, A^{\prime}, \mathcal{K}^{\prime}$ and $\Phi^{\prime}$ defined as in 1.7). Moreover, we claim that $A(\sigma, \kappa ; X, I, \mathcal{A}, \mathcal{K})$ implies $A\left(\sigma, \kappa ; X^{\prime}, I^{\prime}, \mathcal{A}^{\prime}, \mathcal{K}^{\prime}\right)$ and $B(\sigma, \kappa,|X|)$ implies $B\left(\sigma, \kappa ;\left|X^{\prime}\right|\right)$. The latter implication being trivial, let us consider the first.

Now, if $\kappa<\mu<\left|X^{\prime}\right|(\leq|X|)$ and $\operatorname{cf}(\mu) \leq \sigma$ then let us choose $\tau$ and $J$ as in 1.3 for $X, I, A$ and $K$. We claim that $\tau$ and $J^{\prime}=J \cap P\left(X^{\prime}\right)$ will be as required for $X^{\prime}, I^{\prime}, A^{\prime}$ and $K^{\prime}$. However, it is again straightforward to show that the "primed" versions of (b)-(d) in 1.3 follow immediately from their "unprimed" versions and the definitions of $I^{\prime}, A^{\prime}$ and $K^{\prime}$, respectively.

Putting this together with the minimality of $|X|=\lambda$ we get that $\Phi\left(A \uparrow_{I} \quad Y\right.$ ) must be valid for every $Y \in[X]^{<\lambda}$; hence condition (3) of Lemma 1.1 is satisfied. Our aim now is to show that the other conditions of 1.1 are also satisfied. This will of course imply that $\Phi(\mathcal{A})$ is valid, in contradiction to our assumption that $\Phi(\mathcal{A})$ fails, and thus conclude the proof of 1.6.

Let us note that conditions (ii) and (iii) in 1.2 immediately imply that if $Y \subset X$ then

$$
\left|\left\{A \in A: A \cap Y \in \mathcal{K}^{+}\right\}\right| \leq|Y|^{\sigma}
$$


In particular, if $|Y| \leq \kappa$ then, since $\kappa^{\sigma}=\kappa$, we have by (iv) that $\Phi(\{A \in A: A \cap Y \in$ $\left.\mathcal{K}^{+}\right\}$) holds, hence we must have $|X|=\lambda>\kappa$.

In order to apply 1.1 we still have to find a set of cardinals $C$ and a semi-ideal $J$ as required there. As it turns out, the choice of these will depend on $\lambda$ and on whether $A(\sigma, \kappa ; X, I, A, \mathcal{K})$ or $B(\sigma, \kappa, \lambda)$ was assumed; hence we shall have to distinguish cases accordingly.

Case 1. For each $\mu<\lambda$ we have $\mu^{\sigma}<\lambda$. Then we put $C=\left\{\mu<\lambda: \mu^{\sigma}=\mu\right\}$ and $J=K$. Note that in this case $(*)$ immediately implies condition (2) of 1.1 ; hence it remains only to show that condition (1) holds, i.e. that $\hat{A} \cap \cup K \subset I$. Thus let $\left\{F_{\nu}: \nu \in \alpha\right\}$ be an increasing chain of elements of $P(A) \cap \mathcal{K}$ for some $A \in A$, i.e. such that $F_{\nu} \subset F_{\nu^{\prime}}$ if $\nu \in \nu^{\prime} \in \alpha$. Then by (i) from 1.2 each $F_{\nu} \in I$; hence if $\operatorname{cf}(\alpha) \leq \sigma$ then, since $I$ is $\sigma^{+}$-additive, $F=\bigcup\left\{F_{\nu}: \nu \in \alpha\right\} \in I$ as well. If, on the other hand, $\operatorname{cf}(\alpha)>\sigma$, then $[F]^{\sigma}=\bigcup\left\{\left[F_{\nu}\right]^{\sigma}: \nu \in \alpha\right\}$; hence by (iii) in 1.2 we have $F \in \mathcal{K}$; hence by (i) again $F \in \mathcal{K} \cap \hat{A} \subset I$.

Case 2. There is a $\mu<\lambda$ with $\mu^{\sigma} \geq \lambda$ and $A(\sigma, \kappa ; X, I, \mathcal{A}, \mathcal{K})$ holds. Let $\mu_{0}$ be the smallest cardinal $\mu$ with $\mu^{\sigma} \geq \lambda$, then we have $\kappa<\mu_{0}<\lambda$ and $\operatorname{cf}\left(\mu_{0}\right) \leq \sigma$; hence there exists a cardinal $\tau>\sigma$ and a semi-ideal $J$ on $X$ as required in 1.3. In this case we put $C=\left\{\mu: \mu_{0} \leq \mu<\lambda\right\}$ and use $J$ as the semi-ideal needed in 1.1.

Now, condition (d) in 1.3 is simply identical with condition (1) in 1.1; hence only (2) remains to be verified. This is going to be done by induction on $\mu \in C$. To begin with, we show that for any $Y \in[X]^{\mu_{0}},\left|\left\{A \in A: A \cap Y \in J^{+}\right\}\right| \leq \mu_{0}$. To see this, let us note first that we have $\operatorname{cf}\left(\mu_{0}\right) \leq \sigma$; hence $Y$ can be written as an increasing union, $Y=\bigcup\left\{Y_{\nu}: \nu \in \alpha\right\}$, where $\alpha \leq \sigma$ and $\left|Y_{\nu}\right|=\gamma_{\nu}<\mu_{0}$ for all $\nu \in \sigma$. Now if $A \in A$ and $A \cap Y \in J^{+}$then $\alpha \leq \sigma<\tau$ and (c) in 1.3 imply that $A \cap Y_{\nu} \in J^{+}$for some $\nu \in \alpha$; moreover (b) implies that

$$
\left\{A \in A: A \cap Y_{\nu} \in J^{+}\right\} \subset\left\{A \in A: A \cap Y_{\nu} \in K^{+}\right\} .
$$

But then, using $(*)$ again, we get

$$
\left|\left\{A \in A: A \cap Y_{\nu} \in J^{+}\right\}\right| \leq\left|Y_{\nu}\right|^{\sigma}<\mu_{0}
$$

Hence, as we claimed,

$$
\left|\left\{A \in \mathcal{A}: A \cap Y \in J^{+}\right\}\right| \leq \mu_{0} \cdot \alpha=\mu_{0} .
$$

Now, assume that $\mu_{0}<\mu<\lambda$ and for every cardinal $\nu$ with $\mu_{0} \leq \nu<\mu$ and every $Y \in[X]^{\nu}$ we have $\left|\left\{A \in A: A \cap Y \in J^{+}\right\}\right| \leq \nu$. By condition (a) of 1.3 we have $\mu_{0}<\mu<\lambda \leq \mu_{0}^{+\tau}$ which implies that $\mu=\mu_{0}^{+\alpha}$ for some ordinal $\alpha<\tau$; consequently either $\mu$ is a successor (and therefore regular) cardinal or $\operatorname{cf}(\mu)=\operatorname{cf}(\alpha)<\tau$. In other words, we either have $\operatorname{cf}(\mu)=\mu$ or $\operatorname{cf}(\mu)<\tau$.

Let us now take any set $Y \in[X]^{\mu}$ and write $Y=\bigcup\left\{Y_{\alpha}: \alpha \in \operatorname{cf}(\mu)\right\}$ as an increasing union with $\left|Y_{\alpha}\right|<\mu$ for all $\alpha \in \operatorname{cf}(\mu)$. If $\operatorname{cf}(\mu)=\mu$ then, since $\kappa<\mu_{0}<\mu$ and every $A \in A$ has size $\leq \kappa$, we get that $A \cap Y=A \cap Y_{\alpha}$ for some $\alpha \in \mu$; hence $\left|\left\{A \in A: A \cap Y \in J^{+}\right\}\right| \leq \mu$ follows from our inductive assumption.

If, on the other hand, $\operatorname{cf}(\mu)=\gamma<\tau$ then condition (c) from 1.3 implies that if $A \cap Y \in J^{+}$for some $A \in A$ then there is some $\alpha \in \gamma$ with $A \cap Y_{\alpha} \in J^{+}$as well; hence again we get $\left|\left\{A \in A: A \cap Y \in J^{+}\right\}\right| \leq \mu$ immediately from our inductive assumption.

Case 3. There is a $\mu<\lambda$ with $\mu^{\sigma} \geq \lambda$ and $B(\sigma, \kappa, \lambda)$ holds. Note that in this case we must have $\mu^{\sigma}=\mu^{+}=\lambda$, and also, as above, $\operatorname{cf}(\mu) \leq \sigma$. In this case we 
simply put $C=\{\mu\}$ and we shall use $\operatorname{Sp}(\sigma, \kappa, \mu)$ to define the semi-ideal $J$ still needed for the applicability of 1.1. In order to make notation easier we shall put in what follows $X=\lambda$.

Now, let $\left\langle P_{\alpha}: \alpha \in \mu^{+}=\lambda\right\rangle$ be as required in 1.4 , the definition of $\operatorname{Sp}(\sigma, \kappa, \mu)$. Then our semi-ideal $J$ is defined as follows:

$$
J=\left\{H \subset \lambda: \forall \beta<\sup H\left(P_{\beta} \cap \mathcal{K}^{+} \cap P(H)=\varnothing\right)\right\},
$$

i.e. $H$ belongs to $J$ if no subset of $H$ belongs to $P_{\beta} \cap K^{+}$for any $\beta<\sup H$. It is clear that $J$ is a semi-ideal and $K \subset J$.

Next we show that condition (1) of 1.1, i.e. $\hat{A} \cap(\cup J) \subset I$ is satisfied. In fact, our first aim is to prove the weaker relation $\hat{A} \cap J \subset I$. Assume, indirectly, that $I^{+} \cap \hat{A} \cap J \neq \varnothing$ and let $H \in I^{+} \cap \hat{A} \cap J$ be such that sup $H=\alpha$ is minimal. Since $I$ is $\sigma^{+}$-complete and $|H| \leq \kappa$ it clearly follows that $\sigma<\operatorname{cf}(\alpha) \leq \kappa$. Then, by $\operatorname{Sp}(\sigma, \kappa, \lambda)$, we can write $H$ in the form

$$
H=\bigcup\left\{H_{\nu}: \nu \in \sigma\right\},
$$

where $\left[H_{\nu}\right]^{\sigma} \subset \bigcup\left\{P_{\beta}: \beta \in \alpha\right\}$ for all $\nu \in \sigma$.

Using the $\sigma^{+}$-additivity of $I$ we see that there is a $\nu \in \sigma$ such that $H_{\nu} \in I^{+}$. By the minimality of $\alpha$ we then have $\sup H_{\nu}=\alpha$ as well; moreover $H_{\nu} \in \hat{A} \cap I^{+} \subset$ $\hat{A} \cap \mathcal{K}^{+}$and condition (iii) of 1.2 imply that there is a set $Z \in\left[H_{\nu}\right]^{\sigma} \cap \mathcal{K}^{+}$. But then there is some $\beta \in \alpha=\sup H_{\nu}$ with $Z \in P_{\beta}$, implying that $H_{\nu} \in J^{+}$, contradicting $H_{\nu} \subset H \in J$. Consequently, we indeed have $\hat{A} \cap J \subset I$.

Now, to show $\hat{A} \cap(\cup J) \subset I$, we again argue by contradiction. Thus we suppose $H \in I^{+} \cap \hat{A} \cap(\cup J)$ and we also assume that $\alpha=\sup H$ is minimal. Similarly as above, then $\kappa \geq \operatorname{cf}(\alpha)>\sigma$. We can write $H$ in the form of an increasing union

$$
H=\bigcup\left\{H_{\xi}: \xi \in \rho\right\},
$$

where each $H_{\xi} \in P(A) \cap J$ for a fixed $A \in \mathcal{A}$. In particular, then we have $H_{\xi} \in I$ for all $\xi \in \rho$ in view of the above; hence if $\operatorname{cf}(\rho) \leq \sigma$ then $H \in I$ by $\sigma^{+}$-additivity, a contradiction.

Thus we must have $\operatorname{cf}(\rho)>\sigma$; hence

$$
[H]^{\sigma}=\bigcup\left\{\left[H_{\xi}\right]^{\sigma}: \xi \in \rho\right\} .
$$

But now we have $H \in I^{+}$; hence by $\hat{A} \cap J \subset I$, also $H \in J^{+}$, and therefore we must have a $\beta \in \sigma=\sup H$ with $P_{\beta} \cap \mathcal{K}^{+} \cap[H]^{\sigma} \neq \varnothing$. Since the $H_{\xi}$ 's are increasing and $\operatorname{cf}(\rho)>\sigma$ then we also must have a $\xi \in \rho$ such that $\beta \in \sup H_{\xi}$ and $P_{\beta} \cap \mathcal{K}^{+} \cap\left[H_{\xi}\right]^{\sigma} \neq \varnothing$. This, however, would mean that $H_{\xi} \in J^{+}$, which is again impossible.

It now remains only to show that condition (2) of 1.1 holds for $C=\{\mu\}$ and the above $J$. For this it will clearly suffice to prove that $\left|\left\{A \in A: A \cap \alpha \in J^{+}\right\}\right| \leq \mu$ holds for each $\alpha \in \mu^{+}=X$.

Let us fix $\alpha \in \mu^{+}$and pick any $A \in A$ with $A \cap \alpha \in J^{+}$. Then, by the definition of $J$, there is some $\beta \in \sup (A \cap \alpha) \leq \alpha$ and a set $Z_{A}$ such that $Z_{A} \in \mathcal{P}_{\beta} \cap \mathcal{K}^{+} \cap[A \cap \alpha]^{\sigma}$. Since $A$ is $K$-a.d. we have $Z_{A} \neq Z_{B}$ if $A \neq B$; hence clearly

$$
\left|\left\{A \in A: A \cap \alpha \in J^{+}\right\}\right| \leq\left|\bigcup\left\{P_{\beta}: \beta \in \alpha\right\}\right| \leq \mu .
$$


Thus we have shown that our Main Lemma 1.1 applies in all three cases; hence $\Phi(A)$ is valid, yielding a contradiction that proves the Main Theorem.

We close this section by giving some conditions that imply the, admittedly rather peculiar looking, assumption $\operatorname{Sp}(\sigma, \kappa, \mu)$ involved in the formulation of $B(\sigma, \kappa, \lambda)$.

1.8. THEOREM. If $\mu$ and $\sigma$ are infinite cardinals such that $\operatorname{cf}(\mu) \leq \sigma<\mu, \square_{\mu}$ holds and $\lambda^{\sigma} \leq \mu$ whenever $\lambda<\mu$ then $\operatorname{Sp}(\sigma, \kappa, \mu)$ holds for all $\kappa \leq \mu$.

PROOF. Let us start by recalling that since $\mu$ is singular, $\square_{\mu}$ can be formulated as follows: with every limit ordinal $\alpha \in \mu^{+}$we can associate a closed unbounded subset $C_{\alpha}$ of $\alpha$ in such a way that

(i) if $\beta \in C_{\alpha}^{\prime}$ (i.e. $\beta$ is a limit point of $C_{\alpha}$ ) then $C_{\beta}=\beta \cap C_{\alpha}$;

(ii) $\left|C_{\alpha}\right|<\mu$.

Since $\operatorname{cf}(\mu) \leq \sigma$ we can write $\mu=\sum\left\{\mu_{\nu}: \nu \in \sigma\right\}$ where $\mu_{\nu}<\mu$ for each $\nu \in \sigma$; moreover each $\alpha \in \mu^{+}$can be written as $\alpha=\bigcup\left\{B_{\nu}^{\alpha}: \nu \in \sigma\right\}$, with $\left|B_{\nu}^{\alpha}\right| \leq \mu_{\nu}$ for $\nu \in \sigma$. Next, for every limit ordinal $\alpha \in \mu^{+}$we put

$$
A_{\nu}^{\alpha}=\bigcup\left\{B_{\nu}^{\beta}: \beta \in C_{\alpha}\right\}
$$

Note that $\left|A_{\nu}^{\alpha}\right| \leq\left|C_{\alpha}\right| \cdot \mu_{\nu}<\mu$. Moreover, if $\beta \in C_{\alpha}^{\prime}$ then $A_{\nu}^{\beta} \subset A_{\nu}^{\alpha}$ for every $\nu \in \sigma$.

Now we define the sequence $\left\langle P_{\alpha}: \alpha \in \mu^{+}\right\rangle$as follows: if $\alpha$ is a successor ordinal we put $P_{\alpha}=\varnothing$; if $\alpha$ is limit then we put $P_{\alpha}=\bigcup\left\{\left[A_{\nu}^{\alpha}\right]^{\sigma}: \nu \in \sigma\right\}$. Since $\left|A_{\nu}^{\alpha}\right|<\mu$ we have, by our assumption, $\left|\left[A_{\nu}^{\alpha}\right]^{\sigma}\right| \leq \mu$; hence $\left|P_{\alpha}\right| \leq \mu$ for all $\alpha$.

Next, if $\alpha \in \mu^{+}$and $\operatorname{cf}(\alpha)>\sigma$ then we have $\alpha=\bigcup\left\{A_{\nu}^{\alpha}: \nu \in \sigma\right\}$; moreover, for each $\nu \in \sigma$,

$$
A_{\nu}^{\alpha}=\bigcup\left\{A_{\nu}^{\beta}: \beta \in C_{\alpha}^{\prime}\right\}
$$

where $\beta, \gamma \in C_{\alpha}^{\prime}$ and $\beta<\gamma$ implies $A_{\nu}^{\beta} \subset A_{\nu}^{\gamma}$. This implies that, since $\operatorname{cf}(\alpha)>\sigma$,

$$
P_{\alpha}=\bigcup\left\{P_{\beta}: \beta \in C_{\alpha}^{\prime}\right\}
$$

Now, if $A$ is an arbitrary subset of $\alpha$ then we can write $A=\bigcup\left\{A \cap A_{\nu}^{\alpha}: \nu \in \sigma\right\}$, and we have

$$
\left[A \cap A_{\nu}^{\alpha}\right]^{\sigma} \subset\left[A_{\nu}^{\alpha}\right]^{\sigma} \subset P_{\alpha} \subset \bigcup\left\{P_{\beta}: \beta \in \alpha\right\}
$$

for every $\nu \in \sigma$, which is clearly much more than required by $\operatorname{Sp}(\sigma, \kappa, \mu)$.

As an immediate corollary of the above result we get the following sufficient condition for $B(\sigma, \kappa, \lambda)$ to hold.

1.9. COROLlaRY. If $\kappa^{\sigma}=\kappa$ and for every cardinal $\mu$ with $\kappa<\mu<\lambda$ and $\operatorname{cf}(\mu) \leq \sigma$ we have $\mu^{\sigma}=\mu^{+}$and $\square_{\mu}$ then $B(\sigma, \kappa, \lambda)$ is valid.

2. Splitting strongly almost disjoint families. The aim of this section is to apply our Main Theorem 1.6 to show that certain strongly almost disjoint families are "essentially disjoint". These results turn out to be generalizations and/or strengthenings of results of Miller [8], Komjáth [7], Erdős and Hajnal [4].

Let us start by introducing some notation and terminology. Given a cardinal $\kappa$, a family $A \subset[X]^{\kappa}$ is said to be $\kappa$-essentially disjoint (in short $\kappa$-e.d.) if to every $A \in A$ we can assign a subset $F(A) \in[A]^{<\kappa}$ in such a way that the family $\{A \backslash F(A): A \in A\}$ is disjoint. We are going to write $\operatorname{ED}(\kappa, \sigma ; \lambda)$ to denote the following statement: Whenever $|X| \leq \lambda$ and $A \subset[X]^{\kappa}$ is $\sigma$-a.d. then $A$ is $\kappa$-e.d. Moreover, $\operatorname{ED}(\kappa, \sigma)$ means that $\operatorname{ED}(\kappa, \sigma ; \lambda)$ holds for all cardinals $\lambda$. 
Let us start by formulating a result which is clearly the basis for the applicability of our Main Theorem to strongly almost disjoint families.

2.1. THEOREM. If $\kappa=\kappa^{\sigma}$ and $A \subset[X]^{\kappa}$ is $\sigma$-a.d., then the quintuple $\left\langle X,[X]^{<\kappa}\right.$, $\left.A,[X]^{<\sigma}, \kappa-\mathrm{ED}(X)\right\rangle$ is $(\sigma, \kappa)$-good. (Here $\kappa-\mathrm{ED}(X)$ denotes the collection of all $\kappa$ e.d. subfamilies of $[X]^{\kappa}$.)

ProOF. Since $\kappa^{\sigma}=\kappa$ implies $\sigma<\operatorname{cf}(\kappa)$ it follows that $[X]^{<\kappa}$ is a $\sigma^{+}$-additive ideal on $X$. Clearly, $[X]^{<\sigma}$ is a semi-ideal for which (i)-(iii) of Definition 1.2 are satisfied. It is also straightforward to check that $\kappa-\operatorname{ED}(X)$ is both $[X]^{<\kappa}$-additive and $[X]^{<\kappa}$-hereditary. Hence the only thing left to show is that if $B \in[A] \leq \kappa$ then $B$ is $\kappa$-e.d. In fact, if $\prec$ is a well-ordering of $B$ of order type $\leq \kappa$ then the $\sigma$-almost disjointness of $B$ clearly implies that, for any $A \in B$,

$$
F(A)=\bigcup\{B \in B: B \prec A\} \cap A
$$

is of cardinality less than $\kappa$; moreover the family $\{A \backslash F(A): A \in B\}$ is disjoint. This completes the proof of 2.1 .

Now we are ready to formulate the main results of this section.

2.2. THEOREM. If $\kappa=\kappa^{\sigma} \geq \omega$ then $\operatorname{ED}\left(\kappa, \sigma ; \kappa^{+\omega}\right)$ holds.

ProOF. Assume that $|X| \leq \kappa^{+\omega}$ and $A \subset[X]^{\kappa}$ is $\sigma$-a.d. Now, if $\kappa<\mu<|X|$ then $\mu=\kappa^{+n}$ for some positive natural number $n$; hence $\operatorname{cf}(\mu)=\mu>\sigma$. Thus $A\left(\sigma, \kappa ; X,[X]^{<\kappa}, \mathcal{A},[X]^{<\sigma}\right)$ and $B(\sigma, \kappa,|X|)$ are both trivially satisfied. Consequently, from 2.1 and the Main Theorem we immediately conclude that $A$ is $\kappa$-e.d.

We shall see later that 2.2 is sharp in the sense that, modulo some large cardinals, the failure of $\operatorname{ED}\left(\kappa, \sigma ; \kappa^{+\omega+1}\right)$ can be consistent. On the other hand, our next results show that some reasonably mild set-theoretic assumptions enable us to replace $\kappa^{+\omega}$ with larger cardinals in 2.2 .

2.3. DEFInItION. If $\sigma, \kappa, \lambda$ are cardinals then $A(\sigma, \kappa ; \lambda)$ denotes the following statement: $\kappa^{\sigma}=\kappa$ and for every cardinal $\mu$, if $\kappa<\mu<\lambda$ and $\operatorname{cf}(\mu) \leq \sigma$ then there is a regular cardinal $\tau<\kappa$ such that $\min \left\{\mu^{\sigma}, \lambda\right\} \leq \mu^{+\tau}$. Moreover, $A(\sigma, \kappa)$ means that $A(\sigma, \kappa ; \lambda)$ is valid for all $\lambda$.

Note that if $\kappa=\kappa^{\sigma} \geq \omega$ then $A\left(\sigma, \kappa ; \kappa^{+\omega}\right)$ is trivially valid; hence if $\sigma^{+}<\kappa$ then 2.3 is an immediate consequence of our next result.

2.4. THEOREM. If $\sigma^{+}<\kappa$ then $A(\sigma, \kappa ; \lambda)$ implies $\operatorname{ED}(\kappa, \sigma, \lambda)$. In particular $\sigma^{+}<\kappa$ and $A(\sigma, \kappa)$ imply $\operatorname{ED}(\kappa, \sigma)$.

Proof. Of course, given $X$ with $|X| \leq \lambda$ and $A \subset[X]^{\kappa}$ that is $\sigma$-a.d., we start again by considering the $(\sigma, \kappa)$-good quintuple $\left\langle X,[X]^{<\kappa}, A,[X]^{<\sigma}, \kappa-\operatorname{ED}(X)\right\rangle$ and show that it satisfies the condition $A\left(\sigma, \kappa ; X,[X]^{<\kappa}, \mathcal{A},[X]^{<\sigma}\right)$. Then the Main Theorem implies that $A$ is $\kappa$-e.d.

Thus we consider any cardinal $\mu$ with $\kappa<\mu<|X| \leq \lambda$ and $\operatorname{cf}(\mu) \leq \sigma$; by $A(\sigma, \kappa, \lambda)$ there is a regular cardinal $\tau<\kappa$ with $\min \left\{\mu^{\sigma}, \lambda\right\} \leq \mu^{+\tau}$. Since $\sigma^{+}<\kappa$ we may also assume that $\sigma<\tau$. Having chosen $\tau$ we take $[X]^{<\tau}$ as the semi-ideal $J$ required in 1.3. It is obvious that $\tau$ and $J=[X]^{<\tau}$ will satisfy conditions (a)-(d) of 1.3: Indeed (a) holds by the choice of $\tau$, (b) holds because $\sigma<\tau$, (c) holds because $\tau$ is regular and (d) holds because $\tau<\kappa$. 
Now, if $\kappa$ is an arbitrary infinite cardinal and $(\sigma=) n \in \omega$ then $A(n, \kappa)$ is trivially valid; hence we immediately obtain the following strengthening of Miller's theorem, which for $\kappa=\omega$ is due to P. Komjáth [7].

2.5. COROLlARY. For every infinite cardinal $\kappa$ and $n \in \omega \operatorname{ED}(\kappa, n)$ holds.

As we shall see later, the assumption $\sigma^{+}<\kappa$ in 2.4 is necessary, i.e. $A\left(\sigma, \sigma^{+}, \lambda\right)$ (or $A\left(\sigma, \sigma^{+}\right)$) does not imply $\operatorname{ED}\left(\sigma^{+}, \sigma, \lambda\right)$ (or $\operatorname{ED}\left(\sigma^{+}, \sigma\right)$ ). Our next result shows that the stronger assumption $B\left(\sigma, \sigma^{+}, \lambda\right)$ does imply $\operatorname{ED}\left(\sigma^{+}, \sigma, \lambda\right)$. Of course, since $B\left(\sigma, \sigma^{+}, \sigma^{+\omega}\right)$ is trivially valid, it also yields the case $\kappa=\sigma^{+}$of 2.2 that was not covered by 2.4 .

2.6. THEOREM. If $\sigma \geq \omega$ then $B\left(\sigma, \sigma^{+}, \lambda\right)$ implies $\operatorname{ED}\left(\sigma^{+}, \sigma, \lambda\right)$; therefore $B\left(\sigma, \sigma^{+}\right)$implies $\operatorname{ED}\left(\sigma^{+}, \sigma\right)$.

Proof. Assume $B\left(\sigma, \sigma^{+}, \lambda\right)$ and let $A \subset[X]^{\sigma^{+}}$be $\sigma$-a.d. with $|X| \leq \lambda$. Then we may directly apply our Main Theorem to $\left\langle X,[X]^{<\sigma+}, \mathcal{A},[X]^{<\sigma}, \sigma^{+}-\operatorname{ED}(X)\right\rangle$, which is $\left(\sigma, \sigma^{+}\right)$-good by 2.1 , to conclude that $A$ is $\sigma^{+}$-e.d.

Let us finally formulate a consequence of 2.5 and 2.6 which we think is particularly interesting.

2.7. COROLlaRY. Let $\sigma$ be any infinite cardinal. Then $\operatorname{ED}\left(2^{\sigma}, \sigma\right)$ holds if either (i) $2^{\sigma}>\sigma^{+}$and $A\left(\sigma, 2^{\sigma}\right)$ holds or (ii) $2^{\sigma}=\sigma^{+}$and $B\left(\sigma, 2^{\sigma}\right)$ is valid.

The above results all remain valid if the families $A \subset[\lambda]^{\kappa}$ are only assumed to be $\alpha$-a.d. for some ordinal $\alpha$ with $|\alpha|=\sigma$. Of course, by $\alpha$-a.d. we mean that the order-type of the intersection of any two members of $A$ is less than $\alpha$.

3. Splitting families of closed sets. In this section we deduce several topological consequences of our Main Theorem. They will all have the form that certain families of closed subsets of any topological space can be "split simultaneously", i.e., the underlying set can be split in such a way that every member of the family is split by it. Since these families of closed sets are not almost disjoint in any sense, we cannot apply the Main Theorem or its consequences to them directly. In fact, our next result will be the key in introducing some kind of almost disjoint family into the picture.

In order to make the formulation of this lemma easier we introduce the following terminology: Let $A$ and $B$ be families of sets; we say that $A$ supports $B$ if for every $B \in B$ there is an $A \in A$ with $A \subset B$, and $B$ is said to cover $A$ if for every $A \in A$ there is a $B \in B$ such that $A \subset B$.

3.1. LemMA. Let $X$ be a set, $I$ an ideal and $\mathcal{K}$ a semi-ideal on $X$ with $K \subset I$; moreover $\mathcal{L}$ and $R$ are subfamilies of $I^{+}$satisfying conditions (1)-(3) below:

(1) $R$ supports $\mathcal{L}$;

(2) if $R \in R$ and $L \in \mathcal{L}$ then $R \cap L \in \mathcal{K}$ or $R \cap L \in I^{+}$;

(3) for each $R \in R$ there is a $K$-a.d. family $A(R) \subset P(R) \cap I^{+}$that supports $\{R \cap L: L \in \mathcal{L}\} \cap I^{+}$.

Then there is a $\mathcal{K}$-a.d. family $A \subset I^{+}$that supports $\mathcal{L}$ and is covered by $R$.

ProOF. Let $B$ be a maximal $K$-a.d. subfamily of $R$ and put

$$
A=\bigcup\{A(R): R \in B\} \text {. }
$$


We claim that $A$ is as required. It is immediate from the choice of $A$ that $A \subset I^{+}$, $A$ is $K$-a.d., and that $R$ covers $A$. To see that $A$ supports $\mathcal{L}$, take any $L \in \mathcal{L}$ and note that there is some $B \in B$ such that $L \cap B \in I^{+}$. Indeed, otherwise we had $L \cap B \in \mathcal{K}$ for all $B \in B$ and since by (1) there is some $R \in R$ with $R \subset L$ we had $R \notin B$ but $R \cap B \in \mathcal{K}$ for all $B \in B$, contradicting the maximality of $B$. Thus we have some $A \in A(B)$ with $A \subset L \cap B \subset L$, which was to be shown.

Before we formulate our next result we introduce some more notation and terminology. A family $A$ of subsets of $X$ is said to be $\kappa$-colorable (in symbols: $\operatorname{col}_{\kappa}(A)$ ) if there is a map $p: X \rightarrow \kappa$ such that for every $A \in A$ one has $p[A]=\kappa$. In other words, $\operatorname{col}_{\kappa}(A)$ means that the points of $X$ can be colored by $\kappa$ colors in such a way that every element of $A$ has all the possible colors. Note that $\operatorname{col}_{2}(A)$ is the same as saying that $A$ has property $B$.

Let $\sigma, \kappa, \lambda$ be cardinals. Then $\operatorname{COL}_{\gamma}(\kappa, \sigma ; \lambda)$ denotes the following statement: Every $\sigma$-a.d. family $A \subset[\lambda]^{\kappa}$ is $\gamma$-colorable. We also write $\operatorname{COL}_{\gamma}(\kappa, \sigma)$ to denote that $\mathrm{COL}_{\gamma}(\kappa, \sigma ; \lambda)$ is valid for all $\lambda$. Let us note here that $\operatorname{ED}(\kappa, \sigma ; \lambda)$ obviously implies $\mathrm{COL}_{\kappa}(\kappa, \sigma ; \lambda)$; hence the results of $\S 2$ yield conditions under which $\mathrm{COL}_{\kappa}(\kappa, \sigma ; \lambda)$ (or $\left.\mathrm{COL}_{\kappa}(\kappa, \sigma)\right)$ is valid.

3.2. THEOREM. Let $\sigma$ and $\kappa$ be cardinals satisfying $\kappa^{\sigma}=\kappa, X$ an arbitrary topological space and $\mathcal{L}$ a family of closed sets in $X$ such that if $L \in \mathcal{L}$ then $|L| \geq \kappa$; moreover for every closed subset $F$ of $L$ we have either $|F|<\sigma$ or $|F| \geq \kappa$. Then $\mathrm{COL}_{\gamma}(\kappa, \sigma,|X|)$ implies $\operatorname{col}_{\gamma}(\mathcal{L})$.

ProOF. Let us put $I=[X]^{<\kappa}$ and $\mathcal{K}=[X]^{<\sigma}$; then we have $\mathcal{L} \subset I^{+}$. We also define a family $R \subset I^{+}$such that 3.1 could be applied.

Let us say that a set $Y \in[X]^{\kappa}$ is $\kappa$-homogeneous if every set $H$ closed in the subspace $Y$ satisfies $|H|<\sigma$ or $|H|=\kappa$. We choose $R$ to be the collection of all $\kappa$-homogeneous subsets of $X$.

Next we show that conditions (1)-(3) of 3.1 hold. First, to show that $R$ supports $\mathcal{L}$ consider $L \in \mathcal{L}$ and fix for every $H \in[L]^{\sigma}$ a set $F(H) \in[L]^{\kappa}$ with $H \subset F(H) \subset$ $\bar{H}$. Then, by transfinite recursion on $\alpha \in \sigma^{+}$we define sets $Y_{\alpha} \subset L$ as follows: Let $Y_{0} \in[L]^{\kappa}$ be arbitrary. If $\alpha \in \sigma^{+} \backslash\{0\}$ and we have already defined $Y_{\beta}$ for all $\beta \in \alpha$ then we put

$$
Y_{\alpha}=\bigcup\left\{F(H): H \in\left[\bigcup_{\beta \in \alpha} Y_{\beta}\right]^{\sigma}\right\} .
$$

It is easy to check that then $R=\bigcup_{\alpha \in \sigma^{+}} Y_{\alpha} \in R$, because if $H \in[R]^{\sigma}$ then we have $H \subset Y_{\alpha}$ hence

$$
|\bar{H} \cap R|=\left|\bar{H} \cap Y_{\alpha+1}\right|=\kappa
$$

for some $\alpha \in \sigma^{+}$. Moreover we also have $R \subset \bar{Y}_{0} \subset L$, since $L$ is closed; hence $R$ supports $\mathcal{L}$.

Next, if $L \in \mathcal{L}$ and $R \in R$ then $L \cap R$ is closed in $R$; hence $L \cap R \in \mathcal{K}$ or $L \cap R \in I^{+}$by the definition of $\kappa$-homogeneity. This implies that (2) is valid.

Finally, to check (3) let us first note that if $L \cap R \in I^{+}$then in fact $L \cap R \in R$; hence if $H \in[L \cap R]^{\sigma}$ is arbitrary then $\bar{H} \cap R=\bar{H} \cap L \cap R \in R$ as well. This means that the family of sets closed in $R$ and having a dense set of size $\sigma$ supports the family $\{L \cap R: L \in \mathcal{L}\} \cap I^{+}$; hence it suffices to find a $\mathcal{K}$-a.d. $A(R) \subset P(R) \cap I^{+}$ that supports the former. However, $\kappa^{\sigma}=\kappa$ implies that there are at most $\kappa$ many 
sets closed in $R$ with a dense set of size $\sigma$; hence it is well known that we may even have a disjoint family $A(R) \subset P(R) \cap I^{+}$that supports these sets.

Thus we can apply 3.1 now and obtain a $\sigma$-a.d. family $A \subset[X]^{\kappa}$ that supports $\mathcal{L}$. Then $\mathrm{COL}_{\gamma}(\kappa, \sigma,|X|)$ implies that $\mathcal{A}$ is $\gamma$-colorable; hence clearly so is also $\mathcal{L}$.

As an immediate corollary of 3.2 and 2.4 we obtain the next result, which is a significant improvement of (10.1) from [3]. It is based on the following well-known fact (see e.g. [6]): If $X$ is an infinite compact Hausdorff space with no nontrivial convergent sequences then $|X| \geq 2^{\omega_{1}}$. Since $\beta \omega$ is such a space and $|\beta \omega|=2^{2^{\omega}}$ we see that if $\kappa_{0}$ is the minimum cardinality of a compact $T_{2}$ space with no convergent sequences then $2^{\omega_{1}} \leq \kappa_{0} \leq 2^{2^{\omega}}$.

If $X$ is an arbitrary topological space then we denote by $S(X)$ the family of all closed subspaces of $X$ that are infinite, compact $T_{2}$ and contain no convergent sequences.

3.3. THEOREM. If $X$ is a topological space, $\kappa=\kappa^{\omega} \leq \kappa_{0}$ and $\mathrm{COL}_{\gamma}(\kappa, \omega,|X|)$ holds, then $S(X)$ is $\gamma$-colorable. In particular, $A\left(2^{\omega_{1}}, \omega\right)$ or if $\kappa_{0}^{\omega}=\kappa_{0}$ then even $A\left(\kappa_{0}, \omega\right)$ implies that for any space $X$ we have $\operatorname{col}_{2} \omega_{1}(S(X))$ or $\operatorname{col}_{\kappa_{0}}(S(X))$, respectively.

In view of the above it would be of some interest to know whether $\kappa_{0}^{\omega}=\kappa_{0}$ is always valid, but this question seems to remain open.

Another direct application of 3.2 could be obtained from the following result of Malychin: If $K$ is a compact $T_{1}$ space of countable pseudocharacter then $|K| \leq \omega$ or $|K| \geq 2^{\omega}$. In particular this would imply certain results about splitting the family $C(X)$ of all closed Cantor sets in a space $X$. We do not, however, bother to write these down because applying our Main Theorem rather than 3.2 we get a much stronger result.

To this end, let us first list a few definitions and facts. A subset $R$ of a space $X$ is called relatively countably closed (in short r.c.c.) if for every countably infinite subset $A \subset R$ if $A$ has a limit point in $X$ it also has one in $R$. Clearly, if $F \subset X$ is closed and $R \subset X$ is r.c.c. then $F \cap R$ is also r.c.c. The following easy lemma taken from [3] will play an essential role below.

3.4. LEMMA. Suppose that $Y$ is a $T_{1}$ space, $f$ is a closed and continuous map of $Y$ into the Cantor set $\mathbf{C}$ and $R \subset Y$ is r.c.c. Then $f[R]$ is closed in $\mathbf{C}$.

PROOF. If $f[R]$ is not closed then we can choose a sequence $\left\{y_{n}: n \in \omega\right\} \subset f[R]$ of pairwise distinct elements that converges to a point $y \notin f[R]$. Let us pick for each $n \in \omega$ a point $x_{n} \in R$ such that $f\left(x_{n}\right)=y_{n}$. Then the set $A=\left\{x_{n}: n \in \omega\right\}$ cannot be closed in $Y$ since the map $f$ is closed and $f[A]=\left\{y_{n}: n \in \omega\right\}$ is not closed. Since $R$ is r.c.c. there is a limit point $x$ of $A$ in $R$. We claim that $f(x)=y$. Indeed, since $f$ is continuous, $Y$ is $T_{1}$, every neighborhood of $x$ contains infinitely many $x_{n}$ 's; hence $f(x)$ is a limit point of $\left\{y_{n}: n \in \omega\right\}$, i.e. $f(x)=y$. Thus we arrived at a contradiction with $y \notin f[R]$; hence $f[R]$ must be closed.

3.5. THEOREM. Let $X$ be a topological space with $|X|=\lambda$, and let $\mathcal{L}(X)$ denote the family of all closed $T_{1}$ subspaces of $X$ that admit a closed and continuous map onto C. Then $\mathcal{L}(X)$ is $2^{\omega}$-colorable if either (a) $\lambda \leq\left(2^{\omega}\right)^{+\omega}$, or (b) $\omega_{1}<2^{\omega}$ and $A\left(\omega, 2^{\omega}, \lambda\right)$ holds, or $(\mathrm{c}) B\left(\omega, 2^{\omega}, \lambda\right)$ holds. 
ProOF. For each $L \in \mathcal{L}(X)$ let us fix a closed continuous onto map $f_{L}: L \rightarrow \mathbf{C}$ and put $\mathcal{F}=\left\{f_{L}: L \in \mathcal{L}(X)\right\}$. We then define an ideal $I$ on $X$ by stipulating $A \in I$ if and only if $|f[A]|<2^{\omega}$ for all $f \in \mathcal{F}$. Since $\operatorname{cf}\left(2^{\omega}\right)>\omega$, it is clear that $I$ is countably (i.e. $\omega_{1^{-}}$) additive. Next we define a (semi-)ideal $\mathcal{K}$ on $X$ by putting $A \in \mathcal{K}$ if $|\overline{f[A]}| \leq \omega$ (i.e. the closure of $f[A]$ in $\mathbf{C}$ is countable) for all $f \in \mathcal{F}$. Obviously, we have $K \subset I$. Finally, we denote by $R$ the collection of all r.c.c. subsets $R$ of $X$ of cardinality $2^{\omega}$ and such that $R \in I^{+}$.

Now we show that Lemma 3.1 can be applied to $\mathcal{L}=\mathcal{L}(X), I, \mathcal{K}$ and $R$. To see that (1) holds, i.e. $R$ supports $\mathcal{L}(X)$, we note first that for every closed $L \subset X$ and for every $A \in[L]^{\omega}$ there is an r.c.c. set $R_{A}$ such that $A \subset R_{A} \subset L$ and $\left|R_{A}\right|=2^{\omega}$. Indeed, to get such an $R_{A}$ we have to start out with $A$ and then add limit points to countable sets (that have any) iteratively $\omega_{1}$ times. Now, if $L \in \mathcal{L}(X)$ we may choose $A \in[L]^{2}$ in such a way that $f_{L}[A]=\mathbf{C}$ and then the set $R_{A}$ constructed as above will clearly belong to $I^{+}$, hence to $R$ as well.

Next, to check (2), take $L \in \mathcal{L}(X)$ and $R \in R$ such that $L \cap R \notin \mathcal{K}$. This means that there is an $M \in \mathcal{L}(X)$ such that $\overline{f_{M}[L \cap R]}$ is uncountable. However, $M \cap L \cap R$ is clearly r.c.c. in $M$; hence by $3.4, f_{M}[L \cap R]=f_{M}[M \cap L \cap R]$ is closed in $\mathbf{C}$; hence according to the above it must have cardinality $2^{\omega}$. But this means that $L \cap R \in I^{+}$. Let us note that we have actually shown that if $L \cap R \notin \mathcal{K}$ then $L \cap R \in R$.

Finally, to verify (3), let us first note that for every $R \in R$ there is $R_{1} \in R$ which is separable and closed in $R$. Indeed, let $f \in \mathcal{F}$ be such that $|f[R]|=2^{\omega}$ and $S \subset f[R]$ be a countable dense set in $f[R]$. It is quite straightforward to check that if $Z$ is a countable subset of $R$ such that $f[Z]=S$ then the closure $R_{1}$ of $Z$ in $R$ is r.c.c. in $X$; hence by $3.4 f\left[R_{1}\right]$ is closed in $\mathbf{C}$ and since $S \subset f\left[R_{1}\right] \subset f[R]$ we must have $f\left[R_{1}\right]=f[R]$. This shows, of course, that $R_{1} \in R$.

In particular, if $L \in \mathcal{L}(X)$ and $L \cap R \in I^{+}$(hence $L \cap R \in R$ ) then $L \cap R$ contains a separable member of $R$ that is closed in $L \cap R$ and thus in $R$ as well. Consequently it will suffice to show that there is a family $A(R) \subset P(R) \cap I^{+}$which is $K$-a.d. (in fact it will be disjoint) and supports the family of separable closed subsets of $R$ belonging to $R$.

It is quite easy, however, to construct such a family because $|R|=2^{\omega}$ implies that there are at most $2^{\omega}$ many separable closed subsets of $R$. (Indeed, this makes use of the same idea as Bernstein's original result.)

What we actually show is that if $B$ is any subfamily of $I^{+}$with $|B| \leq 2^{\omega}$ then with every $B \in B$ we can associate a subset $F(B) \subset B$ such that $F(B) \in I^{+}$and $F\left(B_{1}\right) \cap F\left(B_{2}\right)=\varnothing$ if $B_{1} \neq B_{2}$. To see this, let us first fix for each $B \in B$ a function $g_{B} \in \mathcal{F}$ such that $\left|g_{B}[B]\right|=2^{\omega}$ and then consider an enumeration $\left\langle B_{\alpha}: \alpha \in 2^{\omega}\right\rangle$ of $B$ such that for each $B \in B$ we have

$$
\left|\left\{\alpha \in 2^{\omega}: B_{\alpha}=B\right\}\right|=2^{\omega} .
$$

We also put $g_{\alpha}=g_{B_{\alpha}}$ for $\alpha \in 2^{\omega}$.

Now, by transfinite recursion on $\alpha \in 2^{\omega}$ we define points $p_{\alpha} \in X$ as follows. If $\alpha \in 2^{\omega}$ and $p_{\beta}$ has been picked for all $\beta \in \alpha$, then since $\left|g_{\alpha}\left[B_{\alpha}\right]\right|=2^{\omega}$ we can pick a point $p_{\alpha} \in B_{\alpha} \backslash\left\{p_{\beta}: \beta \in \alpha\right\}$ such that $g_{\alpha}\left(p_{\alpha}\right) \neq g_{\alpha}\left(p_{\beta}\right)$ for every $\beta \in \alpha$. Having completed the recursion, we put for $B \in B$

$$
F(B)=\left\{p_{\alpha}: B=B_{\alpha}\right\}
$$


It is clear from the above construction that $F(B) \subset B$ and if $B_{1} \neq B_{2}$ then $F\left(B_{1}\right) \cap F\left(B_{2}\right)=\varnothing$; moreover we have $\left|g_{B}[F(B)]\right|=2^{\omega}$; hence $F(B) \in I^{+}$.

We have now checked that conditions (1)-(3) of Lemma 3.1 are valid; hence there is a $K$-a.d. family $A \subset I^{+}$that supports $\mathcal{L}(X)$ and is covered by $R$. Since $|R|=2^{\omega}$ for $R \in R$ this clearly implies that $A \subset[X]^{2^{\omega}}$.

Let $\Phi$ be the following property of subfamilies $B$ of $P(X)$ : for each $B \in B$ there is $F(B) \subset B$ with $F(B) \in I^{+}$such that $F\left(B_{1}\right) \cap F\left(B_{2}\right)=\varnothing$ if $B_{1} \neq B_{2}$. Note that $\Phi$ is obviously $I$-additive and $I$-hereditary; moreover we have just established that $\Phi(B)$ holds for each $B \in\left[I^{+}\right] \leq 2^{\omega}$.

We now claim that the quintuple $\langle X, I, \mathcal{A}, \mathcal{K}, \Phi\rangle$ is $\left(\omega, 2^{\omega}\right)$-good. Since $I, \mathcal{A}, \mathcal{K}$ and $\Phi$ are the right types of objects, moreover conditions (i) and (ii) of 1.2 are obviously satisfied, and (iv) follows immediately from what we have noted about $\Phi$ above, only (iii) remains to be checked.

Thus let $Y \in K^{+}$; i.e. there is some $f \in \mathcal{F}$ for which $\overline{f[Y]}$ is uncountable. Now, if $S$ is a countable dense subset of $f[Y]$ then there is a countable set $Z \subset Y$ with $f[Z]=S$; hence $\overline{f[Z]}=\bar{S}=\overline{f[Y]}$ is uncountable, showing that $Z \in \mathcal{K}^{+}$; hence (iii) is clearly satisfied.

Now, in cases (a) and (c) we know that $B\left(\omega, 2^{\omega},|X|\right)$ is also valid; hence the Main Theorem immediately implies that $\Phi(A)$ holds. In order to arrive at this conclusion in case (b), we shall show that $A\left(\omega, 2^{\omega} ; X, I, A, \mathcal{K}\right)$ is valid.

Indeed, given a cardinal $\mu$ with $2^{\omega}<\mu<\lambda=|X|$ and $\operatorname{cf}(\mu) \leq \sigma$ we may choose by $A\left(\omega, 2^{\omega}, \lambda\right)$ and $\omega_{1}<2^{\omega}$ an uncountable regular cardinal $\tau<2^{\omega}$ such that $\min \left\{\mu^{\sigma}, \lambda\right\} \leq \mu^{+\tau}$. Then we define a (semi-)ideal $J$ on $X$ as follows:

$$
J=\{H \subset X: \forall f \in \mathcal{F}(|f[H]|<\tau)\} .
$$

Then $\mathcal{K} \subset J \subset I$ is obvious from $\omega<\tau<2^{\omega}, \cup_{<\tau} J \subset J$ holds because $\tau$ is regular, and $\cup J \subset I$ follows from $\tau<2^{\omega}$. Thus $\tau$ and $J$ satisfy conditions (a)-(d) of 1.3 , showing that $A\left(\omega, 2^{\omega} ; X, I, \mathcal{A}, \mathcal{K}\right)$ is valid; hence by the Main Theorem $\Phi(\mathcal{A})$ is valid.

It is obvious, however, that, since $[X]^{<2^{\omega}} \subset I$, every disjoint subfamily of $I^{+}$is $2^{\omega}$-colorable, and since $\mathcal{A}$ supports $\mathcal{L}(X)$ this implies that $\mathcal{L}(X)$ is also $2^{\omega}$-colorable.

Since every countably compact Uryson space with no isolated points has a closed subset that has a closed and continuous map onto $\mathbf{C}$ (see e.g. [3]), we obtain the following result (a slight strengthening of (8.4) from [3]) as an immediate corollary of 3.5.

3.6. CoROllaRY. For any space $X$, if either (i) $\omega_{1}<2^{\omega}$ and $A\left(\omega, 2^{\omega},|X|\right)$ or (ii) $B\left(\omega, 2^{\omega},|X|\right)$ holds then the family of closed, countably compact and dense in itself Uryson subspaces of $X$ is $2^{\omega}$-colorable. Note that this family includes all closed compact $T_{2}$ subspaces with no isolated points.

Comparing this corollary with the case $\sigma=\omega$ of 2.7 we see that $\operatorname{ED}\left(2^{\omega}, \omega\right)$, hence $\mathrm{COL}_{2^{\omega}}\left(2^{\omega}, \omega\right)$, and say " $\operatorname{col}_{2^{\omega}}(C(X))$ for all $X$ " are both valid under the same assumptions. This raises the problem whether e.g. $\operatorname{COL}_{2^{\omega}}\left(2^{\omega}, \omega\right)$ and the statement that " $\operatorname{col}_{2^{\omega}}(C(X))$ holds for all $X$ " are equivalent. In fact, our next result shows that the latter statement actually implies the former.

3.7. THEOREM. Let $2 \leq \gamma \leq 2^{\omega}$ and suppose that $C(X)$ is $\gamma$-colorable for every compact $T_{1}$ space $X$. Then $\mathrm{COL}_{\gamma}\left(2^{\omega}, \omega\right)$ holds. 
ProOF. Let $A \subset[X]^{2^{\omega}}$ be $\omega$-a.d., where we may assume without any loss of generality that $X=\bigcup A$. Let us fix for every $A \in A$ a Cantor set topology $\tau_{A}$ on $A$; i.e. $\tau_{A}$ is such that $\left\langle A, \tau_{A}\right\rangle$ is homeomorphic to C. Next we define a topology on $X$ by stipulating that $F \subset X$ be closed if and only if $F=X$ or $F$ can be written as a finite union $F=F_{1} \cup \cdots \cup F_{n}$ of sets $F_{i}$ closed in some $\left\langle A_{i}, \tau_{A_{i}}\right\rangle$ with $A_{i} \in A$. It is straightforward to check that this way we indeed get a topology on $X$ such that every $\tau_{A}$ for $A \in A$ coincides with the subspace topology on $A$ and $A$ is also closed in $X$. The fact that every two different members of $\mathcal{A}$ have a finite intersection is essentially used here. It is also easy to see that $X$ is compact and $T_{1}$. Thus by our assumption and $A \subset C(X)$ we get that $A$ is $\gamma$-colorable.

The reader should note that the method of proof of 3.7 is very general and can be applied to yield converses of results obtained from 3.2. Let us just mention two such results.

3.8. THEOREM. If $\kappa_{0}=\kappa_{0}^{\omega}$, then $\mathrm{COL}_{\gamma}\left(\kappa_{0}, \omega\right)$ is equivalent to the following: for every (compact $\left.T_{1}\right)$ space $X$ we have $\operatorname{col}_{\gamma}(S(X))$.

3.9. THEOREM. The following are equivalent:

(1) $\mathrm{COL}_{\gamma}\left(2^{2^{\omega}}, \omega\right)$

(2) for every (compact $T_{1}$ ) space $X$ the family $B(X)$ of all closed copies of $\beta \omega$ in $X$ is $\gamma$-colorable.

These results are significant because, in view of the independence results of the next section, they show that some set-theoretic assumptions in results like 3.3 or 3.5 are necessary. However, it would be topologically much more interesting to find counterexamples of this type which are at least Hausdorff (without insisting, of course, that they be compact). We believe this to be possible, though probably not very easy. Let us formulate one such problem explicitly.

3.10. Problem. Is it consistent with ZFC (or with ZFC+GCH) that there is a Hausdorff space $X$ such that $C(X)$ is not 2-colorable?

4. Independence results. The aim of this section is to show that some of the results of the previous sections are not provable in ZFC. The set-theoretically initiated reader will not be surprised to see that these results, necessarily requiring the negation of statements of the form $A(\sigma, \kappa, \lambda)$ or $B(\sigma, \kappa, \lambda)$, involve certain large cardinals.

Before turning to the actual consistency results, however, we formulate several observations that will justify the choice of methods used to produce these counterexamples.

Let $\Phi$ be any property of families of sets. In accordance with our earlier practice we write $\Phi(\kappa, \sigma, \lambda)$ to denote the statement that if $A \subset[\lambda]^{\kappa}$ is $\sigma$-a.d. then $\Phi(A)$. We shall say that " $\Phi$ goes up" if $\Phi(A)$ implies $\Phi(B)$ whenever $A$ supports $B$. $\Phi$ is called additive if it satisfies the following condition: If $S$ is a disjoint collection of sets and for every $S \in S$ we have a family $A_{S} \subset P(S)$ with $\Phi\left(A_{S}\right)$ then we also have $\Phi\left(\bigcup\left\{A_{S}: S \in S\right\}\right)$. Note that, for instance, $\gamma$-colorability or $(\kappa$-)disjointifiability go up and are additive.

4.1. THEOREM. Suppose that $\Phi$ is additive and goes up; moreover $\kappa, \sigma, \lambda$ are cardinals such that for every $\mu<\lambda$ we have $\Phi(\kappa, \sigma, \mu)$. Then $\neg \Phi(\kappa, \sigma, \lambda)$ implies that there exists a stationary subset $S \subset \lambda$ and a $\sigma$-a.d. family $\left\{A_{\alpha}: \alpha \in S\right\}$ such 
that $\bigcup A_{\alpha}=\alpha$ and $\operatorname{tp} A_{\alpha}=\kappa$ for each $\alpha \in S$. In particular, this implies that $\operatorname{cf}(\lambda)>\operatorname{cf}(\kappa)$.

ProOF. Let $A \subset[\lambda]^{\kappa}$ be $\sigma$-a.d. such that $\Phi(\mathcal{A})$ fails. Since $\Phi$ goes up, we may clearly assume that $\operatorname{tp} A=\kappa$ for every $A \in A$. Let us now put

$$
S=\{\bigcup A: A \in A\}
$$

it will obviously suffice to show that $S$ is stationary in $\lambda$.

Thus assume that $C \subset \lambda$ is closed unbounded and $C \cap S=\varnothing$. Let $C=\left\{\alpha_{\nu}\right.$ : $\nu \in \tau\}$ be the increasing enumeration of $C$ and for every $\nu \in \tau$ put

$$
A_{\nu}=\left\{A \backslash \alpha_{\nu}: A \in A \& \alpha_{\nu}<\sup A<\alpha_{\nu+1}\right\}
$$

Then for all $\nu \in \tau$ we have $\Phi\left(A_{\nu}\right)$ by our assumption; hence $\Phi\left(\bigcup\left\{A_{\nu}: \nu \in \tau\right\}\right)$ because $\Phi$ is additive. But $\bigcup\left\{A_{\nu}: \nu \in \tau\right\}$ supports $\mathcal{A}$; consequently we would also have $\Phi(\mathcal{A})$ since $\Phi$ goes up, a contradiction.

Since 2-colorability (i.e. property $B$ ) is the weakest "splitting" property used in practice, hence its negation is the strongest among the negations of such properties, we may consider the next result as a kind of converse to 4.1 .

4.2. THEOREM. Let $S$ be a stationary subset of $\lambda$ satisfying $\diamond(S)$ and let $\left\{A_{\alpha}: \alpha \in S\right\}$ be a family of infinite sets such that $A_{\alpha} \subset \alpha$ for all $\alpha \in S$. We may then find for every $\alpha \in S$ a set $B_{\alpha} \subset A_{\alpha}$ with $\left|B_{\alpha}\right|=\left|A_{\alpha}\right|$ such that $\left\{B_{\alpha}: \alpha \in S\right\}$ is not 2-colorable.

Proof. Let $\left\{S_{\alpha}: \alpha \in S\right\}$ be a $\diamond(S)$-sequence and with its help we define $B_{\alpha}$ for $\alpha \in S$ as follows:

$$
B_{\alpha}= \begin{cases}A_{\alpha} \cap S_{\alpha} & \text { if }\left|A_{\alpha} \cap S_{\alpha}\right|=\left|A_{\alpha}\right| \\ A_{\alpha} \backslash S_{\alpha} & \text { otherwise }\end{cases}
$$

Clearly we have $\left|A_{\alpha}\right|=\left|B_{\alpha}\right|$ for all $\alpha \in S$.

Now let $X \subset \lambda$ be arbitrary and $\alpha \in S$ be such that $X \cap \alpha=S_{\alpha}$. Clearly, we must then have either $B_{\alpha} \subset S_{\alpha} \subset X$ or $B_{\alpha} \subset \alpha \backslash S_{\alpha} \subset \lambda \backslash X$, which shows that $\left\{B_{\alpha}: \alpha \in S\right\}$ is not 2-colorable.

Note that if $\left\{A_{\alpha}: \alpha \in S\right\}$ is $\sigma$-a.d. (i.e. such that $\left|A_{\alpha} \cap A_{\beta}\right|<\sigma$ if $\alpha \neq \beta$ ) then so is $\left\{B_{\alpha}: \alpha \in S\right\}$.

Our topological applications of the independence results (cf. 3.7-3.9) require $\omega$ a.d. families. As we shall see, 4.2 can be used to obtain an $\omega$-a.d. family of sets of size $\omega_{1}$; however to find analogous families consisting of sets of larger size we shall need a different approach, based on the following simple observation.

4.3. THEOREM. Let $\left\{A_{\alpha}: \alpha \in 2^{\lambda}\right\}$ be a family of infinite subsets of $\lambda$. Then we can choose for each $\alpha \in 2^{\lambda}$ a set $B_{\alpha} \subset A_{\alpha}$ with $\left|B_{\alpha}\right|=\left|A_{\alpha}\right|$ in such a way that $\left\{B_{\alpha}: \alpha \in 2^{\lambda}\right\}$ is not 2-colorable.

Proof. Let $\left\{X_{\alpha}: \alpha \in 2^{\lambda}\right\}$ enumerate $P(\lambda)$. Then for every $\alpha \in 2^{\lambda}$ we define $B_{\alpha}$ by stipulating

$$
B_{\alpha}= \begin{cases}A_{\alpha} \cap X_{\alpha} & \text { if }\left|A_{\alpha} \cap X_{\alpha}\right|=\left|A_{\alpha}\right| \\ A_{\alpha} \backslash X_{\alpha} & \text { otherwise. }\end{cases}
$$

It is immediate to check that $\left\{B_{\alpha}: \alpha \in 2^{\lambda}\right\}$ is as required.

An immediate consequence of 4.3 is the following result obtained originally by Miller in [8], using a different method. 
4.4. COROLlaRY. There is an $\omega$-a.d. subfamily of $[\omega]^{\omega}$ which is not 2-colorable.

Proof. Indeed, we need only to apply 4.3 to any $\omega$-a.d. family $A \subset[\omega]^{\omega}$ of cardinality $2^{\omega}$.

In order to get another corollary we first recall a result of Baumgartner [1] which says that it is consistent to have arbitrarily large regular cardinals $\kappa$ such that an $\omega$-a.d. family $A \subset[\kappa]^{\kappa}$ with $|A|=2^{\kappa}$ exists. Applying 4.3 to this $A$ we immediately get the following.

4.5. COROLLARY. It is consistent to have arbitrarily large regular cardinals $\kappa$ such that $\mathrm{COL}_{2}(\kappa, \omega, \kappa)$ fails.

Of course, if $A \subset[\kappa]^{\kappa}$ is $\omega$-a.d. then we have $\kappa^{\omega} \geq|A|$; hence in Baumgartner's above model we must have $2^{\kappa}=\kappa^{\omega}>\kappa$. This sheds some light on the importance of the assumption $\kappa^{\sigma}=\kappa$ in the results of $\S 2$.

It seems to be a much harder problem to find a cardinal $\lambda$ such that e.g. $\mathrm{COL}_{2}\left(2^{\omega}, \omega, \lambda\right)$ fails. If $2^{\omega}>\omega_{1}$ then by $2.7 A\left(\omega, 2^{\omega}\right)$ must also fail, which means a strong violation of the singular cardinal hypothesis, hence the consistency of large cardinals. We could not establish the consistency of the existence of a $\lambda$ as above, although we conjecture that it can be done.

The situation is different if we also assume that $2^{\omega}=\omega_{1}$, or even GCH. In this case, again by 2.7 , the first cardinal $\lambda$ for which $\mathrm{COL}_{2}\left(\omega_{1}, \omega, \lambda\right)$ may fail is $\omega_{\omega+1}$; moreover then $\square_{\omega_{\omega}}$ must also fail. Again, this implies that e.g. measurable cardinals exist in some inner models; hence the use of a large cardinal in our next result is essential.

4.6. THEOREM. If $V$ satisfies $G C H$ and $\kappa$ is supercompact in $V$ then there is a generic extension $W$ of $V$ such that

$$
W \vDash G C H \quad \& \neg \operatorname{COL}_{2}\left(\omega_{1}, \omega, \omega_{\omega+1}\right) .
$$

PROOF. Let us put $\lambda=\kappa^{+\omega}$ and then, using that $\kappa$ is $\lambda^{+}$-supercompact, pick a normal ultrafilter $\mathcal{U}$ on $\left[\lambda^{+}\right]^{<\kappa}$. Since $\left(\lambda^{+}\right)^{\omega}=\lambda^{+}$we also may fix a one-to-one $\operatorname{map} G:\left[\lambda^{+}\right]^{\omega} \rightarrow \lambda^{+}$.

Using a result of Solovay and some obvious reflection properties of $\kappa$ (cf. [9 and 11]) we may choose a set $A \in \mathcal{U}$ such that

(i) the map $P \rightarrow \bigcup P$ is one-one on $A$;

(ii) each $P \in A$ is closed under $G$; i.e. if $a \in[P]^{\omega}$ then $G(a) \in P$;

(iii) for each $P \in A$ we have that $P \cap \kappa$ is an inaccessible cardinal and the order type of $P$ is $(P \cap \kappa)^{+\omega+1}$.

Let us put $S_{1}=\{\bigcup P: P \in A\}$. Then $S_{1}$ is stationary. Indeed, if $C \subset \lambda^{+}$were a club disjoint from $S_{1}$ then for every $P \in A$ we could choose some $f(P) \in P$ with $C \cap(f(P), \cup P]=\varnothing$ and then, by the normality of $\mathcal{U}$, we could find $B \subset A$ with $B \in \mathcal{U}$ and some $\alpha \in \lambda^{+}$such that $f(P)=\alpha$ for all $P \in B$. This, however, is impossible because, by (i), $\{\bigcup P: P \in B\}$ is clearly cofinal in $\lambda^{+}$.

Also by (i), for every $\alpha \in S_{1}$ there is a unique $P(\alpha) \in A$ with $\alpha=\bigcup P(\alpha)$. The map $\alpha \mapsto \kappa \cap P(\alpha)$ is clearly regressive on $S_{1}$; hence there is a stationary subset $S \subset S_{1}$ and a cardinal $\kappa^{*}$ such that $\kappa \cap P(\alpha)=\kappa^{*}$ for each $\alpha \in S$.

Next we show that for any two $\alpha, \beta \in S$ we have $|P(\alpha) \cap P(\beta)|<\lambda^{*}=\left(\kappa^{*}\right)^{+\omega}$. Indeed if $Q=P(\alpha) \cap P(\beta)$ had size $\geq \lambda^{*}$ then by (ii) we also had $|Q|=\left|[Q]^{\omega}\right|=$ 
$\left(\lambda^{*}\right)^{\omega}=\left(\lambda^{*}\right)^{+}$, which is impossible because both $P(\alpha)$ and $P(\beta)$ have order type $\left(\lambda^{*}\right)^{+}$but have different suprema.

Let $P$ be the notion of forcing that collapses $\lambda^{*}$ to $\omega$ with finite conditions. Then $V^{\mathcal{P}} \vDash \omega_{1}=\left(\lambda^{*}\right)^{+}$. Let $\mathcal{Q}$ denote the Levy collapse of $\kappa$ to $\omega_{2}$ in $V_{1}=V^{\mathcal{P}}$; it is easy to show that in $V_{2}=\left(V_{1}\right)^{\mathcal{Q}}$ we have GCH; moreover $\omega_{\omega}=\lambda$ and $\omega_{\omega+1}=\lambda^{+}$. Also in this model $\lambda^{*}$ is a countable ordinal and the sequence $\langle P(\alpha): \alpha \in S\rangle$, where $P(\alpha) \in[\alpha]^{\omega_{1}}$ for $\alpha \in S$, satisfies $\operatorname{tp}(P(\alpha) \cap P(\beta))<\lambda^{*}$ for any two $\alpha, \beta \in S$.

Now we can use III, 7.2 of [10] to find a proper poset $R$ such that in $W=V_{2}^{R}$ cardinals are preserved; moreover there is a set $X \in\left[\omega_{1}\right]^{\omega_{1}}$ such that if $a \subset \omega_{1}$ is any element of the ground model $V_{2}$ with tp $a<\lambda^{*}$ then $X \cap a$ is finite. Note that $S$ remains stationary in $W$.

Now, for every $\alpha \in S$ let $h_{\alpha}: \omega_{1} \rightarrow P(\alpha)$ be the increasing enumeration of $P(\alpha)$ in $V_{2}$ (note that the order type of $P(\alpha)$ is $\omega_{1}$ here because of (iii)). Then for every $\alpha \in S$ we define the set $A_{\alpha} \in[\alpha]^{\omega_{1}}$ in $W$ as follows:

$$
A_{\alpha}=h_{\alpha}[X] \text {. }
$$

We claim tht $A_{\alpha} \cap A_{\beta}$ is finite for any two $\alpha, \beta$ in $S$ (i.e. $\left\{A_{\alpha}: \alpha \in S\right\}$ is $\omega$-a.d.). Indeed this follows immediately from

$$
h_{\alpha}^{-1}\left(A_{\alpha} \cap A_{\beta}\right) \subset X \cap h_{\alpha}^{-1}(P(\beta))=X \cap h_{\alpha}^{-1}[P(\alpha) \cap P(\beta)]
$$

where the right-hand side set is finite because $h_{\alpha}^{-1}[P(\alpha) \cap P(\beta)] \in V_{2}$ has order type less than $\lambda^{*}$.

Now, to finish the proof, note that since every $\alpha \in S$ has, in $W$, cofinality $\omega_{1}$, GCH implies that $\diamond(S)$ is valid (cf. [9]); hence by 4.2 there is an $\omega$-a.d. family $\left\{B_{\alpha}: \alpha \in S\right\} \subset\left[\omega_{\omega+1}\right]^{\omega_{1}}$ that is not 2-colorable; hence $\operatorname{COL}_{2}\left(\omega_{1}, \omega, \omega_{\omega+1}\right)$ indeed fails in $W$.

As we have mentioned it in the introduction, 4.6 solves negatively the problem raised by Erdős and Hajnal in [4] whether GCH implies $\mathrm{COL}_{2}\left(\omega_{1}, \omega, \omega_{\omega+1}\right)$. Our next result shows that the $\omega$-a.d. sequence $\left\langle A_{\alpha}: \alpha \in S\right\rangle$ establishing this solution also serves to solve another problem of Erdős and Hajnal from [5].

4.7. THEOREM. Assume GCH. Let $S \subset \omega_{\omega+1}$ be a stationary set of $\omega_{1}$-limits and $\left\langle A_{\alpha}: \alpha \in S\right\rangle$ be such that $\bigcup A_{\alpha}=\alpha, \operatorname{tp} A_{\alpha}=\omega_{1}$ and $\left|A_{\alpha} \cap A_{\beta}\right|<\omega$ if $\alpha, \beta \in S$, $\alpha \neq \beta$. Then there is a graph $E \subset\left[\omega_{\omega+1}\right]^{2}$ on $\omega_{\omega+1}$ of chromatic number $\omega_{2}$ such that $[\omega, \omega]$ does not embed into $E$.

ProOF. Let us put $\kappa=\omega_{\omega+1}$. We may assume that $S=\bigcup\left\{S_{\gamma}: \gamma \in \kappa\right\}$ where the $S_{\gamma}$ 's are disjoint and stationary and $S_{\gamma} \cap(\gamma+1)=\varnothing$ for each $\gamma \in \kappa$. Since GCH holds, we have by [9] that $\diamond\left(S_{\gamma}\right)$ holds for every $\gamma \in \kappa$; thus we can choose for each such $\gamma$ a sequence $\left\langle h_{\alpha}: \alpha \in S_{\gamma}\right\rangle$ such that $h_{\alpha}: \alpha \rightarrow \omega_{1}$ and for any function $h: \kappa \rightarrow \omega_{1}$ the set $\left\{\alpha \in S_{\gamma}: h_{\alpha}=h \uparrow \alpha\right\}$ is stationary in $\kappa$.

For every $\alpha \in S$ we have a unique $\gamma(\alpha) \in \kappa$ with $\alpha \in S_{\gamma(\alpha)}$; clearly we have $\gamma(\alpha)<\alpha$. We are going to put

$$
T_{\gamma}=\bigcup\left\{S_{\delta}: \delta>\gamma\right\}
$$

In order to define the graph $E$ we first define for each $\alpha \in S$ a set $D_{\alpha} \subset \omega_{1}$ as follows:

$$
D_{\alpha}=\left\{\nu \in \omega_{1}: \operatorname{tp}\left(h_{\alpha}^{-1}\{\nu\} \cap T_{\gamma(\alpha)}\right)=\alpha\right\} .
$$


Then we define a map $f_{\alpha}: D_{\alpha} \rightarrow \alpha$ by stipulating that $f_{\alpha}(\nu)$ be equal to the $a(\alpha, \nu)$ th member of $h_{\alpha}^{-1}\{\nu\} \cap T_{\gamma(\alpha)}$, where $a(\alpha, \nu)$ denotes the $\nu$ th element of $A_{\alpha}$.

Next we define, by induction on $\alpha \in S$, sets $E_{\alpha} \subset \alpha$ as follows:

$$
E_{\alpha}= \begin{cases}R\left(f_{\alpha}\right) & \text { if for all } \beta<\alpha E_{\beta} \cap R\left(f_{\alpha}\right) \text { is finite, } \\ \varnothing & \text { otherwise, }\end{cases}
$$

and then put $E=\bigcup\left\{\left[E_{\alpha},\{\alpha\}\right]: \alpha \in S\right\}$; i.e. the vertices less than $\alpha$ and connected to $\alpha$ in $E$ are exactly the elements of $E_{\alpha}$.

It is immediate then that $[\omega: 2]$ does not embed into $E$; moreover since $\beta<\alpha$ and $\{\beta, \alpha\} \in E$ imply $\gamma(\beta)>\gamma(\alpha)$ it follows that there is no infinite increasing path in $E$. It follows then easily that $[\omega, \omega]$ does not embed into $E$ either.

It now remains only to show that $E$ is not $\omega_{1}$-chromatic. Assume, indirectly, that $h: \kappa \rightarrow \omega_{1}$ is a good coloring of $E$. For each $\gamma \in \kappa$ let us put

$$
S_{\gamma}^{\prime}=\left\{\alpha \in S_{\gamma}: h \uparrow \alpha=h_{\alpha}\right\}
$$

and let

$$
N=\left\{\nu \in \omega_{1}:\left|\left\{\gamma:\left|h^{-1}\{\nu\} \cap S_{\gamma}^{\prime}\right|=\kappa\right\}\right|=\kappa\right\} .
$$

It is standard to show that if $\nu \in N$ then

$$
C_{\nu}=\left\{\alpha \in \kappa:(\forall \gamma<\alpha) \operatorname{tp}\left(h^{-1}\{\nu\} \cap T_{\gamma} \cap \alpha\right)=\alpha\right\}
$$

is closed and unbounded in $\kappa$; hence so is $C=\bigcap\left\{C_{\nu}: \nu \in N\right\}$.

On the other hand, if $\nu \in \omega_{1} \backslash N$ then there is a $\gamma_{\nu} \in \kappa$ such that if $\gamma \in \kappa \backslash \gamma_{\nu}$ then $\left|h^{-1}\{\nu\} \cap S_{\gamma}^{\prime}\right|<\kappa$; hence if $\delta=\sup \left\{\gamma_{\nu}: \nu \in \omega_{1} \backslash N\right\}$ then there is a final segment $S^{*}$ of $S_{\delta}^{\prime}$ such that $S^{*} \subset \bigcup\left\{h^{-1}\{\nu\}: \nu \in N\right\}$. Of course, since $S_{\delta}^{\prime}$ is stationary, so is $S^{*}$, and consequently $C \cap S^{*}$ as well.

By tracing back our definitions we see that if $\alpha \in C \cap S^{*}$ then $N \subset D_{\alpha}$ and thus, since $h(\alpha) \in N$ and $h$ is assumed to be a good coloring of $E, \alpha$ cannot be connected to every element of $R\left(f_{\alpha}\right)$; i.e. $E_{\alpha} \neq R\left(f_{\alpha}\right)$. (Of course, we also use here that $h\left\lceil\alpha=h_{\alpha}\right.$.) This implies then that for every $\alpha \in C \cap S^{*}$ there is some ordinal $g(\alpha)<\alpha$ such that $R\left(f_{\alpha}\right) \cap R\left(f_{g(\alpha)}\right)$ is infinite. By the pressing down lemma and a simple counting argument we get that there are a stationary set $Z \subset C \cap S^{*}$, an ordinal $\beta$ and a set $X \in\left[R\left(f_{\beta}\right)\right]^{\omega}$ such that $g(\alpha)=\beta$ and $X \subset R\left(f_{\alpha}\right)$ if $\alpha \in Z$.

But if $\alpha \in Z$ and $x \in X$ then $x=f_{\alpha}(\nu)$ for some $\nu \in D_{\alpha}$ (actually, we must have $\left.\nu=h(x)=h_{\alpha}(x)\right)$, i.e. $x$ is the $a(\alpha, \nu)$ th element of $h_{\alpha}^{-1}\{\nu\} \cap T_{\gamma(\alpha)}=$ $h^{-1}\{\nu\} \cap T_{\delta} \cap \alpha$. But for $\alpha^{\prime} \in Z$ with $\alpha<\alpha^{\prime}$ we get that $x$ is the $a\left(\alpha^{\prime}, \nu\right)$ th element of $h^{-1}\{\nu\} \cap T_{\delta} \cap \alpha^{\prime}$, of which the above set is clearly an initial segment; hence we must have $a(\alpha, \nu)=a\left(\alpha^{\prime}, \nu\right)$. Since $X$ is infinite, this would immediately imply that $A_{\alpha} \cap A_{\alpha \prime}$ is also infinite, a contradiction. Thus the proof of 4.7 is completed.

\section{REFERENCES}

1. J. Baumgartner, Almost-disjoint sets, the dense set problem and the partition calculus, Ann. Math. Logic 9 (1976), 401-439.

2. F. Bernstein, Zur Theorie der Trigonometrischen Reihen, Leipziger Berichte 60 (1908), 325-338.

3. Yu. Bregman, B. Sapirovskiř and A. Sostak, On partitions of topological spaces, Casopis Pěst. Mat. 109 (1984), 27-53.

4. P. Erdős and A. Hajnal, On a property of families of sets, Acta Math. Acad. Sci. Hungar. 12 (1961), 87-124. 
5. __, On chromatic number of graphs and set-systems, Acta Math. Acad. Sci. Hungar. 17 (1966), 61-99.

6. A. Hajnal and I. Juhász, Remarks on the cardinality of compact spaces and their Lindelöf subspaces, Proc. Amer. Math. Soc. 59 (1976), 146-148.

7. P. Komjáth, Families close to disjoint ones, Acta Math. Acad. Sci. Hungar. 43 (1984), 199-207.

8. E. W. Miller, On the property of families of sets, Compt. Rendus Varsovie 30 (1937), 31-38.

9. S. Shelah, On successors of singular cardinals, Proc. Logic Colloq. 1978 (Mons), Stud. Logic Foundations Math., Vol. 97, North-Holland, Amsterdam, 1979.

10. __ Proper forcing, Lecture Notes in Math., vol. 940, Springer-Verlag, Berlin, 1982.

11. R. Solovay, W. N. Reinhardt and A. Kanamori, Strong axioms of infinity and elementary embeddings, Ann. Math. Logic 13 (1978), 73-116.

12. W. Weiss, Partitioning topological spaces, Colloq. Math. Soc. J. Bolyai 23 (1978), 1249 1255.

13. K. Wolfsdorf, Färbungen grosser Würfel..,, Arch. Math. 40 (1983), 569-576.

DePARTMENT OF MATHEMATICS, MATHEMATICAL INSTITUTE OF THE HUNGARIAN ACADEMY OF SCIENCES, BUDAPEST H-1364, HUNGARY (Current address of A. Hajnal and I. Juhász)

Department of Mathematics, The Hebrew UNiversity, JeRUSALEM, ISRAEL (Current of S. Shelah) 